\title{
Reduced Inhibin and Elevated Gonadotropin Levels in Early Pubertal Boys with Testicular Defects
}

\author{
LEO DUNKEL, MARTTI A. SIIMES, AND WILLIAM J. BREMNER \\ Children's Hospital, University of Helsinki, SF-00290 Helsinki, Finland [L.D., M.A.S.], and Veterans Affairs \\ Medical Center, Seattle, Washington 98195 [W.J.B.]
}

\begin{abstract}
In the prepubertal quiescent period, the control of gonadotropin secretion is assumed to be mediated by neuroendocrine inhibitory mechanisms, whereas little or no effect is thought to be mediated by the gonad. However, in pubertal-aged children with primary gonadal failure, gonadotropins increase to supranormal levels, suggesting that gonadal factors become more important in the control of gonadotropin secretion at that age. These gonadal factors have been poorly characterized so far. To clarify the relationship of inhibin and testosterone to the control of gonadotropin secretion during sexual maturation, we examined serum inhibin, testosterone, $\mathrm{LH}$, and FSH concentrations in $\mathbf{1 0}$ boys with testicular defects and in nine healthy boys, in prepuberty and in early puberty. Serum was obtained at 15 -min intervals for at least $6 \mathrm{~h}$ during the night. Prepubertal-aged boys with testicular defects had only slightly higher FSH levels than healthy boys, and their LH and inhibin levels were no different. In contrast, pubertal-aged boys with testicular defects had higher LH and FSH levels and lower inhibin levels than healthy boys. In prepubertal-aged boys, no correlation was found between individual mean inhibin level and mean FSH or LH level. In contrast, in pubertal-aged boys, an inverse nonlinear relationship between mean inhibin level and mean FSH and LH level was seen. The inverse value for inhibin correlated with FSH $(r=0.74, p<0.01)$ and with LH $(r=0.81, p<0.01)$. In conclusion, our results suggest that inhibin is a factor involved in the control of gonadotropin secretion in boys, at least in the pubertal period. (Pediatr Res 33: 514-518, 1993)
\end{abstract}

\section{Abbreviations}

$C V$, coefficient of variation

$1 /$ inhibin, inverse value for inhibin

\footnotetext{
Children with primary gonadal failure manifest diphasic hypergonadotropism. Plasma LH and FSH levels reach the adult castrate range during infancy, fall to normal or near-normal values during childhood, and return to clearly supranormal levels at the normal pubertal age (1). Numerous studies have led to the speculation that the stringent control of gonadotropin secretion in childhood results from the development of the inhibitory system(s) mediated by the CNS (1-5). In childhood, the gonads are believed to exert little negative feedback regulation on gonad-

Received September 21, 1992; accepted January 12, 1993.

Correspondence and reprint requests: Dr. L. Dunkel, Children's Hospital, University of Helsinki, SF-00290 Helsinki, Finland.

Supported by the Academy of Finland.
}

otropin levels. At the onset of puberty, the CNS-mediated inhibitory mechanisms become less operative and the gonadotropin levels increase. Clear hypergonadotropism in pubertal-aged children with primary gonadal failure suggests that the inhibitory mechanisms mediated by the gonad become more important (1). However, the gonadal factors responsible for the control of gonadotropin secretion during the peripubertal period have not been fully characterized. LH secretion in early pubertal and midpubertal boys has been shown to be relatively resistant to the acute feedback effects of testosterone (6). Furthermore, the elevation of gonadotropin levels associated with primary gonadal failure is first manifested by elevated FSH $(1,7)$, which in the male is not clearly regulated by gonadal steroids.

Inhibin is a glycoprotein hormone produced by the testis and ovary. It may have an important role in the regulation of pituitary FSH secretion. Both inhibin A and inhibin B have been shown to suppress FSH secretion by rat pituitary cells in vitro $(8,9)$. In addition, FSH suppression by inhibin was demonstrated in vivo when Findlay et al. (10) showed that 31-kD bovine inhibin suppressed FSH in a dose-dependent manner when infused into castrate ewes. However, the role of inhibin in regulating serum levels of FSH in man in vivo has not yet been defined.

The purpose of this study was to clarify the role of inhibin and testosterone in the control of gonadotropin secretion during the peripubertal period. As a model, we used normo- and hypergonadotropic boys, assuming that the hypergonadotropism (due to testicular defects) reflects decreased gonadal inhibitory mechanisms. In addressing this aim, we collected nocturnal profiles of inhibin, testosterone, LH, and FSH concentrations by frequent sampling of plasma in healthy and hypergonadotropic prepubertal and early pubertal boys.

\section{SUBJECTS AND METHODS}

Subjects. Gonadotropin, inhibin, and testosterone secretion was studied in 19 boys (Table 1). Nine of them were regarded as endocrinologically normal. Four were short, normal boys (height -2.0 through $-1.0 \mathrm{SD}$ for calendar age and -1.0 through $0 \mathrm{SD}$ for bone age). Of these four, three were pubertal aged, and they had signs of pubic hair growth and testicular enlargement as proof against constitutional delay of puberty. Furthermore, all four had normal growth hormone (nocturnal growth hormone peaks, $>22 \mathrm{IU} / \mathrm{L}$ ) and gonadotropin secretion. The other five were healthy, average boys by clinical evaluation. Ten boys were studied because of suspicion of testicular defects. Two boys were male pseudohermaphrodites with clearly subnormal testosterone responses in human chorionic gonadotropin stimulation tests. These two boys showed clear penile growth and decrease in plasma sex hormone binding globulin levels after three intramuscular injections of testosterone enanthate given at 4-wk intervals, as proof against androgen resistance. One boy had the Klinefelter syndrome. The remaining seven boys had survived 
INHIBIN VS FSH LEVELS IN PUBERTY

Table 1. Clinical characteristics of boys

\begin{tabular}{|c|c|c|c|c|c|}
\hline Patient no. & $\begin{array}{c}\text { Chronologic } \\
\text { age (y) }\end{array}$ & $\begin{array}{c}\text { Bone age } \\
(y)^{*}\end{array}$ & Diagnosis $\dagger$ & $\begin{array}{l}\text { Pubic hair } \\
\text { stageł }\end{array}$ & $\begin{array}{l}\text { Testis volume } \\
(\mathrm{mL})\end{array}$ \\
\hline \multicolumn{6}{|l|}{ Healthy boys } \\
\hline \multicolumn{6}{|l|}{ Group 1A } \\
\hline 1 & 4.3 & 4.5 & & I & $\leq 2$ \\
\hline 2 & 5.2 & 5.0 & & I & $\leq 2$ \\
\hline 3 & 8.5 & 8.5 & Short stature & I & $\leq 2$ \\
\hline 4 & 10.0 & 10.1 & & I & $\leq 2$ \\
\hline \multicolumn{6}{|l|}{ Group 2A } \\
\hline 5 & 12.4 & 10.0 & Short stature & II & 4 \\
\hline 6 & 12.6 & 10.0 & Short stature & II & 4 \\
\hline 7 & 12.8 & 11.0 & Short stature & II & 4 \\
\hline 8 & 13.0 & 12.0 & & $\hat{I}$ & 4 \\
\hline 9 & 13.6 & 12.1 & & I & 4 \\
\hline \multicolumn{6}{|c|}{ Boys with testicular defects } \\
\hline \multicolumn{6}{|l|}{ Group 1B } \\
\hline 10 & 5.5 & 5.0 & $\mathrm{MPH}$ & I & $\leq 2$ \\
\hline 11 & 7.2 & 6.0 & $47, X X Y$ & I & $\leq 2$ \\
\hline 12 & 10.6 & 10.5 & ALL & I & $\leq 2$ \\
\hline 13 & 11.4 & 12.0 & ALL & I & $\leq 2$ \\
\hline \multicolumn{6}{|l|}{ Group 2B } \\
\hline 14 & 12.4 & 12.5 & ALL & I & $\leq 2$ \\
\hline 15 & 12.4 & 9.8 & MPH & I & $\leq 2$ \\
\hline 16 & 12.5 & 12.5 & ALL & I & $\leq 2$ \\
\hline 17 & 12.6 & 11.5 & ALL & I & $\leq 2$ \\
\hline 18 & 12.9 & 12.3 & ALL & I & $\leq 2$ \\
\hline 19 & 13.2 & 11.5 & ALL & I & $\leq 2$ \\
\hline
\end{tabular}

* Determined by the method of Greulich and Pyle (12).

$\dagger \mathrm{MPH}$, masculine pseudohermaphroditism; ALL, long-term survivor of acute lymphoblastic leukemia.

$\$$ By the method of Tanner (13).
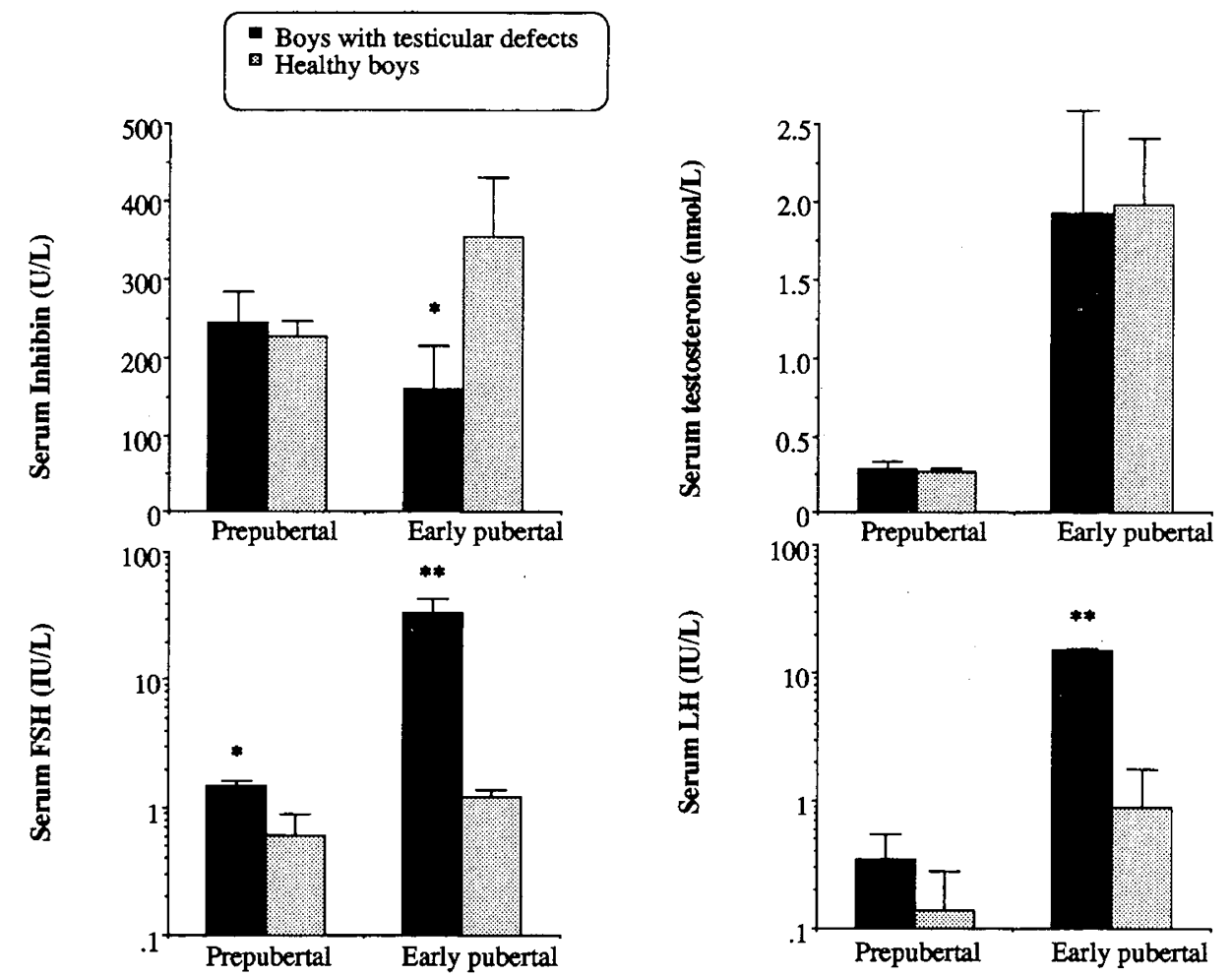

Fig. 1. Mean (+SEM) nocturnal inhibin, testosterone, FSH, and LH concentrations in boys with testicular defects (black bars) and healthy boys (gray bars). Statistically significant differences from the healthy boys are indicated with asterisks: ${ }^{*}, p<0.05 ;{ }^{* *}, p<0.01$.

acute lymphoblastic leukemia; they were treated according to the Scandinavian protocols (11). Their therapy consisted of several cytotoxic drugs known to be specifically harmful for Sertoli cell development. None of the boys had received CNS irradiation or any other androgen therapy except the above- mentioned trial treatment with testosterone. In boys with testicular defects, there was a clear age-dependent change in gonadotropin levels. Younger boys (5.5-11.4 y old) with testicular defects had plasma FSH levels between 1 and 2 SD above the mean for healthy prepubertal control values (7), and older boys 

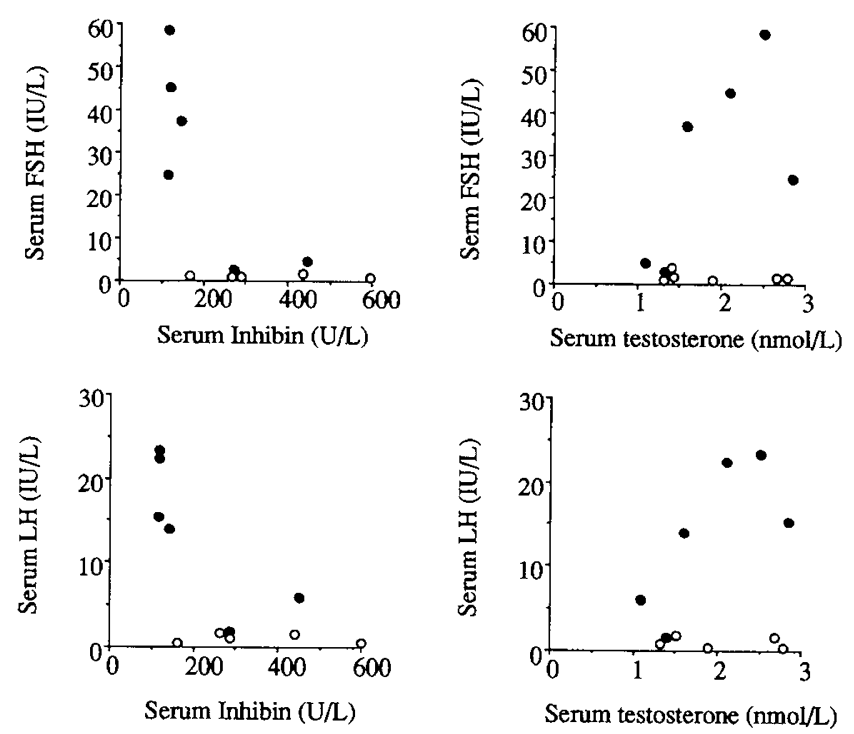

Fig. 2. Correlation of serum gonadotropin levels with inhibin and with testosterone levels in pubertal-aged boys. Healthy boys are represented by open circles and boys with testicular defects as filled circles. Each point on a plot represents the mean value of 24-40 nocturnal samples obtained from each boy.

(12.4-13.2 y old) with testicular defects had plasma FSH and LH levels greater than $3 \mathrm{SD}$ above the mean for healthy early pubertal boys. Because the study was aimed at clarifying mechanisms responsible for the development of the hypergonadotropism in boys with testicular defects at the age puberty should normally occur, we grouped the patients with testicular defects according to both age and gonadotropin levels. Thus, healthy prepubertal boys (testicular volume $\leq 2 \mathrm{~mL}$ ) were compared with the younger prepubertal-aged boys with testicular defects, and healthy early pubertal boys (testicular volumes $=4 \mathrm{~mL}$ ) were compared with the older early pubertal-aged boys with testicular defects. Testicular volumes were measured with Prader's orchidometer. Bone age was determined by the method of Greulich and Pyle (12). Pubertal development was estimated from pubic hair distribution by the method of Tanner (13).

Protocol. The studies were conducted at the Children's Hospital. Informed consent was obtained from a parent and assent from the patient. The protocol was approved by the ethical committee of the hospital.

On the study day, the boys were allowed normal activity from rising until $2100 \mathrm{~h}$. At that time, they went to bed and the room lights were turned off. Sleep was monitored visually by trained nursing personnel. An indwelling i.v. cannula was inserted 30 min before the beginning of sampling, and blood samples were taken at 15 -min intervals from 15 boys for $6 \mathrm{~h}$ during the night and from the remaining three boys for $24 \mathrm{~h}$. Serum LH, FSH, inhibin, and testosterone concentrations were measured in all samples. All the samples from one individual were analyzed in the same assay.

Hormone measurements. Serum inhibin was measured with a heterologous double antibody RIA, using an antiserum to purified $31-\mathrm{kD}$ bovine follicular fluid inhibin and the same antigen labeled with ${ }^{125} \mathrm{I}$ as a tracer as described previously $(14,15)$. Bovine activin-A, Mullerian inhibitory substance, and porcine and human transforming growth factor- $\beta$ had less than $1 \%$ crossreactivity in this assay, as did free $\alpha$ - and $\beta$-subunits of inhibin obtained by reduction and alkylation of $31-\mathrm{kD}$ inhibin. Recently, an $\alpha$-subunit-derived dimeric protein (termed pro- $\alpha-\mathrm{C}$ ) has been isolated from bovine follicular fluid (16). This substance reacts in this inhibin RIA but shows no inhibin-like bioactivity. Whether pro- $\alpha-C$ or other cross-reacting substances occur in humans, and, if so, whether they circulate in blood in significant quantity are unknown. Studies of the the ratio of bioactivity to immunoactivity in women (14) and rats (17) suggest that any interference of nonbioactive substances in the present RIA is small. The sensitivity of the assay was $100 \mathrm{U} / \mathrm{L}$, and the $\mathrm{ED}_{50}$ was $660 \mathrm{U} / \mathrm{L}$. The intraassay $\mathrm{CV}$, based on repeated measurements of multiple dilutions of serum samples covering the bound to free ratio range $40-90 \%$, were $5.8 \%, 3.4 \%$, and $1.8 \%$ in the upper, middle, and lower portions of the working range, respectively. The interassay CV was $10.2 \%$ from 14 assays.

Serum testosterone was measured by RIA after separation of steroid fractions on a Lipidex-5000 microcolumn (Pharmacia, Uppsala, Sweden) as described previously (18). Serum LH and FSH concentrations were measured by time-resolved immunofluorometric assays using reagents from Pharmacia Wallac (Turku, Finland) as described previously (19). The LH standards were calibrated against WHO IRP 68/40, and FSH standards against the 2 nd IRP of pituitary FSH/LH $(78 / 549)$. The assay sensitivity for $\mathrm{LH}$ was $0.019 \mathrm{IU} / \mathrm{L}$ and for $\mathrm{FSH}, 0.014 \mathrm{IU} / \mathrm{L}$, as defined by mean +2 SD of 12 replicates of a 0 sample. For FSH, the $\mathrm{CV}$ ranged from $2.1 \%$ (at $64 \mathrm{IU} / \mathrm{L}$ concentration) to $8.5 \%$ (at $0.1 \mathrm{IU} / \mathrm{L}$ concentration), and for $\mathrm{LH}$, from $3.1 \%$ (at $50 \mathrm{IU} /$ $\mathrm{L}$ concentration) to $13.9 \%$ (at $0.1 \mathrm{IU} / \mathrm{L}$ concentration). The interassay $\mathrm{CV}$, from 12 different assays, ranged from 3.6 to $4.1 \%$ for $\mathrm{FSH}$ and from 5.4 to $5.6 \%$ for $\mathrm{LH}$ at concentrations of approximately 20 and $5 \mathrm{IU} / \mathrm{L}$, respectively.

Pulse analysis. Testosterone, inhibin, LH, and FSH pulse analysis was performed by using a computerized pulse analysis program, Munro (Zaristow software, East Lothian, Scotland), as previously described (7). The program identifies secretory peaks by height and duration from a smoothed baseline, using the assay $\mathrm{SD}$ as a scale factor. The program is essentially an adaptation of the Pulsar program developed by Merriam and Wachter (20).

Statistical analyses. Because of positive skewness of intraindividual $\mathrm{LH}, \mathrm{FSH}$, and testosterone levels, mean values were calculated after logarithmic transformation. Differences between the groups were analyzed by $t$ test. $p$ levels less than 0.05 were considered significant.

\section{RESULTS}

Mean nocturnal inhibin, testosterone, LH, and FSH levels. In healthy boys, mean nocturnal levels of inhibin and testosterone increased with age and development. Such increase was not observed in inhibin levels in boys with testicular defects; inhibin levels between the two groups of boys with testicular defects were not different (Fig. 1). In early puberty, boys with testicular defects had lower inhibin levels than healthy boys, whereas in prepuberty, inhibin levels between the two groups were no different. In healthy boys, individual mean nocturnal gonadotropin levels were very low in prepuberty and they also increased with age and with pubertal development. Prepubertal-aged boys with testicular defects had inhibin, testosterone, and LH levels similar to those in healthy boys; only their FSH levels were slightly higher (Fig. 1). In contrast, early pubertal-aged boys with testicular defects had lower mean inhibin and higher mean gonadotropin levels than healthy boys. Only testosterone levels were similar between the two groups of early pubertal boys.

Inhibin and testosterone levels versus gonadotropin levels. Individual mean nocturnal inhibin concentrations (i.e. mean of 24-40 nocturnal samples from each boy) was correlated with mean individual LH and FSH levels. In prepubertal-aged boys, no correlation was found between individual mean inhibin level and mean FSH or LH level (data not shown). In contrast, in early pubertal-aged boys, an inverse nonlinear relationship between mean inhibin level and mean FSH and LH level was found (Fig. 2). The correlation coefficient between individual mean $1 /$ inhibin and individual mean FSH was $0.74(p<0.01)$ and between individual mean 1 /inhibin and individual mean $\mathrm{LH}$, $0.81(p<0.01)$.

In prepubertal boys, no correlation was found between indi- 

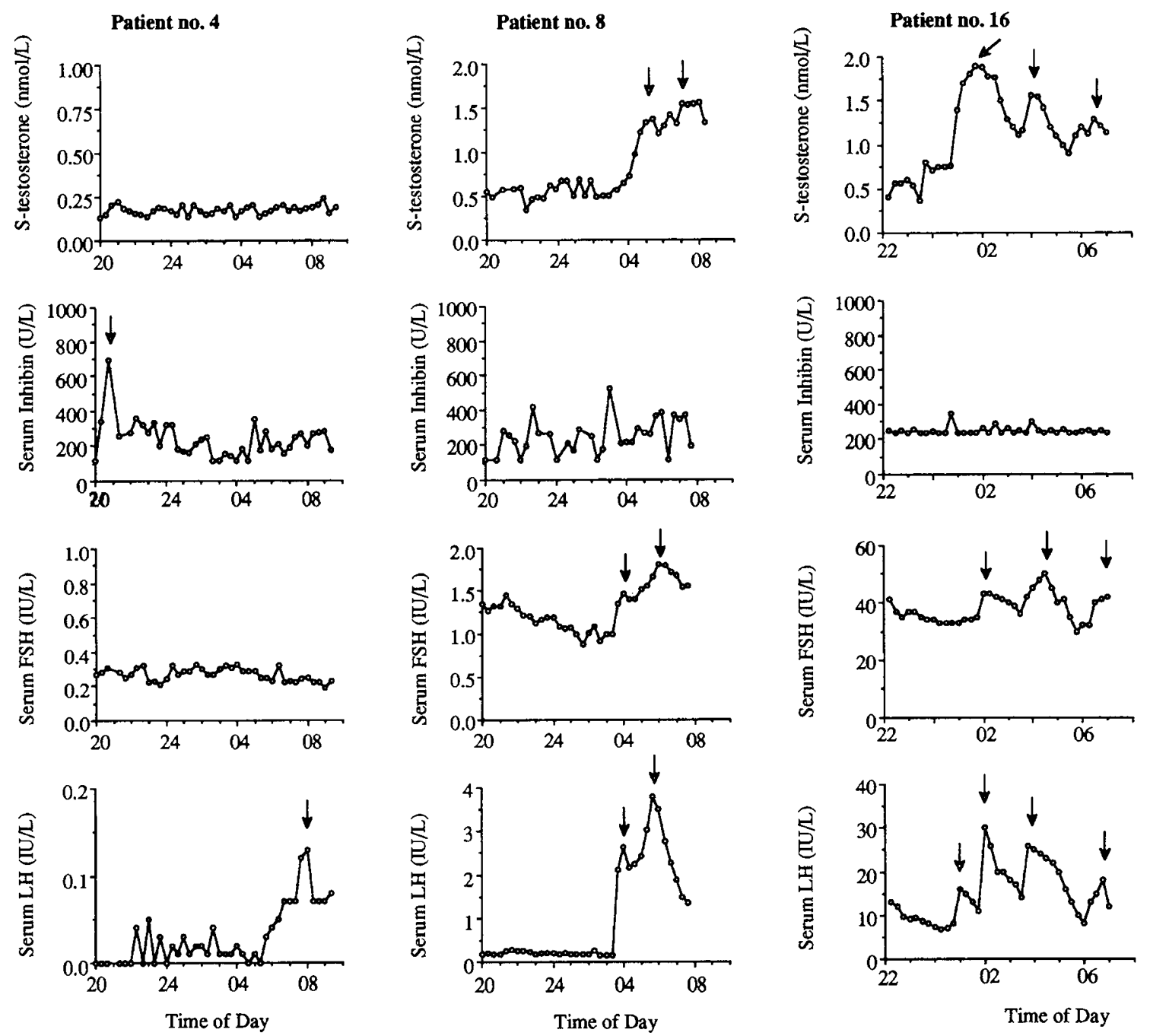

Fig. 3. Time courses of serum testosterone, inhibin, FSH, and LH concentrations in a healthy prepubertal boy (patient 4, left panels), in a healthy early pubertal boy (patient 8 , middle panels), and in an early pubertal boy with testicular defect (patient 16, right panels). Note different scales on the $y$ axes. Statistically significant pulses are indicated with arrows.

vidual mean testosterone levels and mean FSH or LH levels. The same was true for early pubertal boys (Fig. 2).

Inhibin and testosterone levels versus gonadotropin pulses. All boys had significant FSH and LH pulses. Inhibin concentrations also fluctuated with time (Fig. 3), but in only four healthy boys and two boys with testicular defects were these fluctuations recorded as significant pulses (one to two pulses per subject). Therefore, no analysis of the concordance between inhibin and gonadotropin pulses could be performed. Upon visual inspection, no temporal relationship between FSH and inhibin or $\mathrm{LH}$ and inhibin pulses were noted. Furthermore, no association between periods of low inhibin concentration and gonadotropin pulses was observed. Because only a few inhibin pulses were detected overall, we could only study the associations between individual mean inhibin levels and different gonadotropin pulse parameters. In prepubertal boys, individual mean inhibin concentrations did not correlate with any of the various gonadotropin pulse parameters: pulse amplitude, pulse area, pulse number, or preceding pulse nadir. No correlation between mean inhibin and $\mathrm{LH}$ or FSH pulse amplitude, pulse area, or pulse number was found in early pubertal-aged boys. In contrast, in pubertal-aged boys, a significant correlation between individual mean 1 /inhibin and gonadotropin nadir levels preceding a pulse was seen (for 1/ inhibin versus FSH: $r=0.61, p<0.05$; for 1 /inhibin versus $\mathrm{LH}$ : $r=0.85, p<0.001$ ).

In prepubertal-aged boys, testosterone levels did not show any significant individual variation. In contrast, in both groups of early pubertal-aged boys, a small nocturnal increase in plasma testosterone concentration was seen between 0200 and $0500 \mathrm{~h}$ (Fig. 3). All early pubertal-aged boys had at least one significant testosterone pulse. In prepubertal boys, no correlation was found between mean testosterone levels and any of the gonadotropin pulse parameters: pulse amplitude, pulse area, pulse number, or preceding pulse nadir. No correlation was found between individual mean testosterone and any of the gonadotropin pulse parameters in early pubertal-aged boys.

\section{DISCUSSION}

The data of the present study show that among normal and hypergonadotropic boys an inverse relationship exists between mean integrated serum inhibin and FSH levels at an early pubertal age but not in prepuberty. This finding is consistent with the original concept that inhibin regulates the secretion of FSH in a negative feedback fashion. Recently, a similar inverse relationship between inhibin and FSH levels was reported in normal adult men (21), whereas in hypergonadotropic adult men this relationship has not been seen $(21-23)$. The reason for the lack of inverse relationship between serum inhibin and FSH levels in previous studies of adult hypergonadotropic men is unclear, but it may be related to the pulsatile nature of gonadotropin and inhibin secretion. We could observe significant fluc- 
tuation in both FSH and inhibin levels with no temporal relationship when obtaining serum by frequent sampling. Thus, when only single determinations of the hormones are made as in the previous studies (21-23), a considerable amount of information can be lost due to this phenomenon.

We observed that the mean individual inhibin level correlated negatively with the mean individual gonadotropin level. Furthermore, the mean inhibin level correlated negatively with gonadotropin nadir levels but not with gonadotropin pulse number or amplitude. In other words, high inhibin levels were associated with low gonadotropin levels preceding a gonadotropin pulse. This suggests that the inhibitory role of inhibin involves pituitary gonadotropin synthesis and/or release, but inhibin levels do not modulate gonadotropin pulse amplitude or frequency.

The boys with testicular defects displayed a clear age-dependent change in gonadotropin levels. The prepubertal-aged boys with testicular defects had only modest elevation of FSH levels, whereas the pubertal-aged boys had clearly supranormal levels of both gonadotropins. We believe that this difference reflects more operative CNS-mediated inhibition of gonadotropin control in the younger boys and the presence of gonadal inhibitory mechanisms in the the older boys.

We also observed an increase in circulating inhibin with age and sexual maturation in normal boys. This is compatible with the results of a previous study of inhibin concentration during puberty in boys (24). A similar increase during puberty has been reported in the cynomolgus monkey, Macaca fascicularis (25). Inhibin regulation of gonadotropin secretion in vivo has been studied in rodents (26) and primates (27). Both in the rat and in the monkey, immunoneutralization of circulating inhibin results in a selective hypersecretion of FSH, suggesting that inhibin suppresses FSH secretion but not LH secretion in vivo. (26). Intravenous injection of the inhibin-a antiserum caused significant elevations in plasma FSH levels in male rats aged 10-24 d, whereas no significant changes occurred in older animals. This result suggests that endogenous inhibin plays a physiologic role in suppressing FSH secretion in neonatal male rats. However, the data obtained from rodents are not applicable to primates because there are marked differences in the patterns of inhibin secretion during sexual maturation between rodents (26) and primates $(24,25)$. In rats, circulating inhibin levels decline during sexual maturation, whereas in primates, the levels increase. Furthermore, in primates, prepubertal castration leads to little increase in gonadotropins, whereas in rats, gonadotropins rise to the same levels as in adult castrates $(3,4)$. In the present study, inhibin and FSH did not have an inverse relationship in the prepubertal age, as would have been anticipated from the study in rats (26). This was not surprising, inasmuch as overall the gonadotropin levels were very low.

Integrated testosterone concentrations did not show any inverse relationship to either LH or FSH levels. In a previous study, it was shown that amplification of LH secretion during the early hours of sleep in early puberty is relatively resistant to the acute negative feedback effects of testosterone (6). The data of the present study and the previous study suggest that the establishment of the gonadal steroid-mediated negative feedback control on gonadotropin secretion is a relatively late phenomenon in pubertal maturation.

In conclusion, the testis clearly has a role in the control of gonadotropin secretion in the early pubertal period. In normal and hypergonadotropic early pubertal boys, the negative correlation between inhibin and FSH levels supports the concept of inhibin feedback on FSH secretion. Our results suggest that this feedback is operative at least in pubertal-aged boys. Lack of correlation between gonadotropin and testosterone levels sug- gests that the inverse relationship between inhibin and FSH is independent of testosterone.

\section{REFERENCES}

1. Conte FA, Grumbach MM, Kaplan SL 1975 A diphasic pattern of gonadotropin secretion in patients with the syndrome of gonadal dysgenesis. J Clin Endocrinol Metab 40:670-674

2. Kelch RP, Marshall JC, Sauder SE, Hopwood NJ, Reame NE 1983 Neuroendocrine Aspects of Human Reproduction. Academic Press, New York, pp 229-256

3. Plant TM 1988 The Physiology of Reproduction. Raven Press, New York, $1763-1788$

4. Plant TM 1982 Pulsatile luteinizing hormone secretion in the neonatal rhesus monkey (Macaca mulatta). J Endocrinol 93:71-74

5. Marshall J, Kelch RP 1986 Gonadotropin-releasing hormone: role of pulsatile secretion in the regulation of reproduction. N Engl J Med 315:1459-1488

6. Hale PM, Khoury S, Fosters CM, Beitins IZ, Hopwood NJ, Marshall JC, Kelch RP 1988 Increased luteinizing hormone pulse frequency during sleep in early to midpubertal boys: effects of testosterone infusion. J Clin Endocrinol Metab 66:785-791

7. Dunkel L, Alfthan H, Stenman U-H, Perheentupa J 1990 Gonadal control of pulsatile secretion of luteinizing hormone and follicle-stimulating hormone evaluated by ultrasensitive time-resolved immunofluorometric assays. J Clin Endocrinol Metab 70:107-114

8. Robertson DM, Foulds LM, Leversha L 1985 Isolation of inhibin from bovine follicular fluid. Biochem Biophys Res Commun 126:220-226

9. Miyamoto K, Hasegawa Y, Fukuda M 1985 Isolation of porcine follicular fluid inhibin of $32 \mathrm{~K}$ daltons. Biochem Biophys Res Commun 129:396-403

10. Findlay JK, Robertson DM, Clarke IJ 1987 Influence of dose and route of administration of bovine follicular fluid and the suppressive effect of purified 31 kilodalton bovine inhibin on plasma FSH in ovariectomized ewes. J Reprod Fertil 80:455-461

11. Gustafsson G, Garwicz S, Hertz H, Johannesson G, Jonmundsson G, Moe PJ, Salmi T, Seip M, Siimes M, Yssing M, Ảhlström L 1987 A population based study of children with acute lymphoblastic leukemia in the five Nordic countries diagnosed July 1981 through June 1985: incidence, characteristics and treatment results. Acta Paediatr Scand 76:781-788

12. Greulich WW, Pyle SI 1955 Atlas of Skeletal Development of the Hand and Wrist. Stanford University Press, Stanford, CA

13. Tanner JM 1978 Growth at Adolescence. Blackwell, Oxford, UK

14. Robertson DM, Tsonis CG, McLachlan RI 1988 Comparison of inhibin immunological and in vitro biological activities in human serum. J Clin Endocrinol Metab 67:438-443

15. McLachlan RI, Robertson DM, Healy DI, Burger HG, de Kretser DM 1987 Circulating immunoreactive inhibin levels during the normal human menstrual cycle. J Clin Endocrinol Metab 65:954-961

16. Robertson DM, Giacometti M, Foulds LM 1989 Isolation of inhibin alpha subunit precursor proteins from bovine follicular fluid. Endocrinology 125:2141-2149

17. Robertson DM, Hayward S, Irby D 1988 Radioimmunoassay of rat serum inhibin: changes after PMSG stimulation and gonadectomy. Mol Cell Endocrinol 58:1-8

18. Apter D, Jänne O, Karvonen P, Vihko R 1976 Simultaneous determination of five sex hormones in human serum by radioimmunoassay after chromatography on Lipidex-5000. Clin Chem 22:32-38

19. Hemmilä I, Dakubu S, Mukkula V-M, Siitari H, Lövgren T 1984 Europium as a label in time-resolved immunofluorometric assays. Anal Biochem 137:335-343

20. Merriam GR, Wachter KW 1982 Algorithms for the study of episodic hormone secretion. Am J Physiol 243:E310-E318

21. Plymate SR, Paulsen CA, McLachlan RI 1992 Relationship of serum inhibin levels to serum follicle stimulating hormone and sperm production in normal men and men with varicoceles. J Clin Endocrinol Metab 74:859-864

22. De Kretser DM, McLachlan RI, Robertson DM, Burger HG 1989 Serum inhibin in normal men and men with testicular disorders. $J$ Endocrinol 120:517-523

23. Tsatsoulis A, Shalet SM, Robertson DM, Morris ID, Burger HD, De Kretser DM 1988 Plasma inhibin levels in men with chemotherapy induced severe damage to the seminiferous epithelium. Clin Endocrinol (Oxf) 29:659-665

24. Burger HG, McLachlan RI, Bangah M 1988 Serum inhibin concentrations rise throughout normal male and female puberty. J Clin Endocrinol Metab 67:689-694

25. Keeping HS, Winters SJ, Attardi B, Troen P 1990 Developmental changes in testicular inhibin and androgen-binding protein during sexual maturation in cynomolgus monkey, Macaca fascicularis. Endocrinology 126:2858-2867

26. Rivier C, Cajander S, Vaughan J, Hsueh AWJ, Vale W 1988 Age-dependent changes in physiological action, content, and immunostaining of inhibin in male rats. Endocrinology 123:120-126

27. Medhamurthy R, Abeyawardene SA, Culler MD, Negro-Vilar A, Plant T 1990 Immunoneutralization of circulating inhibin in the hypophysiotropically clamped male rhesus monkey (Macaca mulatta) results in a selective hypersecretion of follicle-stimulating hormone. Endocrinology 126:2116-2124 